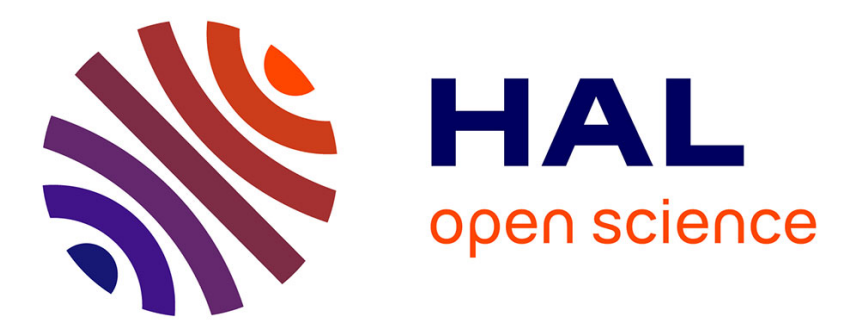

\title{
Model Predictive Control as an Industrially Applicable Approach for Power Control of Solid Oxide Fuel Cells
}

Wiebke Frenkel, Julia Kersten, Harald Aschemann, Andreas Rauh

\section{To cite this version:}

Wiebke Frenkel, Julia Kersten, Harald Aschemann, Andreas Rauh. Model Predictive Control as an Industrially Applicable Approach for Power Control of Solid Oxide Fuel Cells. 2021 25th International Conference on Methods and Models in Automation and Robotics (MMAR), Aug 2021, Międzyzdroje, Poland. pp.251-256, 10.1109/MMAR49549.2021.9528484 . hal-03419472

HAL Id: hal-03419472

https://hal-ensta-bretagne.archives-ouvertes.fr/hal-03419472

Submitted on 22 Nov 2021

HAL is a multi-disciplinary open access archive for the deposit and dissemination of scientific research documents, whether they are published or not. The documents may come from teaching and research institutions in France or abroad, or from public or private research centers.
L'archive ouverte pluridisciplinaire HAL, est destinée au dépôt et à la diffusion de documents scientifiques de niveau recherche, publiés ou non, émanant des établissements d'enseignement et de recherche français ou étrangers, des laboratoires publics ou privés. 


\section{Model Predictive Control as an Industrially Applicable Approach for Power Control of Solid Oxide Fuel Cells}

\author{
Wiebke Frenkel, Julia Kersten and Harald Aschemann \\ Chair of Mechatronics, University of Rostock \\ 18059 Rostock, Germany \\ Email: \{Wiebke.Frenkel, Julia.Kersten, Harald.Aschemann\}@ uni-rostock.de
}

\author{
Andreas Rauh \\ Lab-STICC, ENSTA Bretagne \\ 29806 Brest, France \\ andreas.rauh@interval-methods.de
}

\begin{abstract}
In this paper, a model predictive control (MPC) combined with a discrete-time stationary Kalman filter as an observer for non-measureable states and input disturbances is presented as a simple and industrially applicable approach for controlling the electric power of a solid oxide fuel cell (SOFC). The developed controller was tested in a simulation in terms of its robustness under consideration of model uncertainties and measurement noise. The results were compared with a PI output-feedback controller combined with a feedforward control and an internal model control (IMC). For the MPC the framework conditions are equal to the PI controller and the IMC. Since these conditions can be reproduced by simulations, we can omit a rerun of the experiments. As a result, the MPC provides comparable results and presents as the better of the two alternative controllers.
\end{abstract}

\section{INTRODUCTION}

In view of climatic changes, solid oxide fuel cells (SOFC) become increasingly important by implementing power supply based on renewable energies, if also the supplied fuel gases can be provided in a climatically neutral way. Progressive research into SOFCs produces a lot of new applications, especially as decentralized power stations by using the produced electric and thermal energy simultaneously. In general, electric power is produced in SOFCs due to the internal cold combustion as a result of the redox-reaction

$$
\begin{aligned}
2 \mathrm{H}_{2}+2 \mathrm{O}^{2-} & \rightarrow 2 \mathrm{H}_{2} \mathrm{O}+4 e^{-} \\
\mathrm{O}_{2}+4 e^{-} & \rightarrow 2 \mathrm{O}^{2-} \\
2 \mathrm{H}_{2}+\mathrm{O}_{2} & \rightarrow 2 \mathrm{H}_{2} \mathrm{O}
\end{aligned}
$$

(oxidation at the anode) (reduction at the cathode) (redox-reaction)

inside the stack module. The free electrons, which arise from the reaction of the supplied hydrogen gas with oxygen ions at the anode, shown in Fig. 1, can be used as a directed current source for the consumers. The aim of this paper is to control the resulting power with the methodology of a linear discretetime model predictive control.

In general, state-of-the-art approaches for power controllers of SOFCs are offline generated look-up tables including relations between the electric power, the inlet gas mass flow of hydrogen and the corresponding electric current in terms

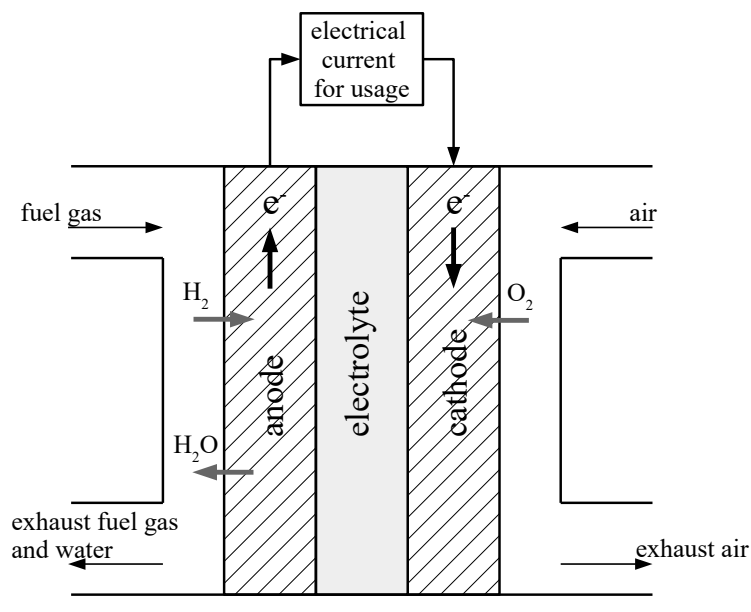

Fig. 1. Schematic representation of the oxidation and reduction processes at both anode and cathode sides of an SOFC.

of a pure open-loop formulation. As already discussed in [1], the development of those look-up tables is a time-consuming process accompanied by innumerable experiments, which are inaccurate, if the operation of the SOFC differs from the experimental conditions. To avoid these inaccurate quasistatic open-loop control approaches, feedback controllers are a promising alternative. For the model predictive control presented in this paper, it was sufficient to perform only a limited number of experiments for analyzing the dynamics of the system and the input/output behavior between the hydrogen gas mass flow and the electric power.

First, in Sec. II, the usually highly nonlinear SOFC system is modeled by a finite-order transfer function approximation based on measured data of previous work [1]. Since an industrially applicable control design should be as simple as possible, we want to provide an easy way for an implementation on a real system. Models based on neural networks, e.g. in [2], [3], are, therefore, less suitable. For the control design, with the aim of keeping the online computational complexity as low as possible, the SOFC system is approximated further 
by a third-order lag system as a simplified representation, which is transformed into its controllable canonical form. Afterwards, the system is augmented by predicted state and output variables to prepare the design of the MPC procedure. The MPC design, described in Sec. III, was performed by solving an optimization problem in a pure offline manner, which results in the gains of a combined feedforward and feedback control by an offline evaluation of the corresponding necessary optimality conditions [4]. Because information about the electric power as well as its temporal derivatives are crucially required, a discrete-time Kalman filter was developed. To further compensate input disturbances, the filter design makes use of an extended state-space representation to handle additive disturbances in a lumped manner. For that reason, an integrator disturbance model was introduced. The simulation results with a nominal process, in Sec. IV, as well as the simulation with a disturbed system mimicking the experiments presented in [1], in Sec. V, of the MPC were compared with the results of a PI controller, which is combined with a dynamic feedforward control, and an internal model control (IMC) of previous work [1]. Finally, Sec. VI gives conclusions and an outlook on future work.

\section{Modeling The SOFC System}

In general, the overall SOFC system can be described by different component models, which consist of the thermal behavior of the SOFC itself and the gas preheaters cf. [5] [8]. The electro-chemical behavior described more detailed in previous work [1], [9] forms the basis for this paper.

Based on measurements from an available test rig, a corresponding transfer function

$$
G_{\mathrm{S}}(s)=\frac{P_{\mathrm{el}}}{\dot{m}_{\mathrm{H}_{2}}}(s) \cdot G_{\mathrm{PT} 1}(s)=\frac{\sum_{i=0}^{5} b_{\mathrm{S}, i} \cdot s^{i}}{\sum_{i=0}^{6} a_{\mathrm{S}, i} \cdot s^{i}} \cdot \frac{1}{T s+1}
$$

was determined by using techniques for transfer function estimation which are based on various measured open-loop step responses [10], which was already validated in [1]. The resulting transfer function includes the relation between the electric power $P_{\mathrm{el}}$ (as the subsystem output) and the hydrogen mass flow $\dot{m}_{\mathrm{H}_{2}}$ (system input) given by sixth-order dynamics as well as the mass flow's first-order lag dynamics $G_{\mathrm{PT} 1}(s)$ related to fluidic inertia.

For the control and observer design, a state-space representation of the system is necessary. As shown in previous work [1], the reduced.order transfer function

$$
G_{\mathrm{S}, \mathrm{PT} 3}(s)=\frac{b_{0}}{s^{2}+a_{1} s+a_{0}} \cdot \frac{1}{T s+1},
$$

i.e., a third-order lag model identified by means of the same measurement data as the more detailed model and the added mass flow's first-order lag dynamics (2), approximates the SOFC process for the power control sufficiently enough. The state-space representation is given by transforming (3) into its controllable canonical form

$$
\dot{\mathbf{x}}=\underbrace{\left[\begin{array}{ccc}
0 & 1 & 0 \\
0 & 0 & 1 \\
-a_{31} & -a_{32} & -a_{33}
\end{array}\right]}_{\mathbf{A}} \cdot \underbrace{\left[\begin{array}{l}
0 \\
0 \\
b
\end{array}\right]}_{\mathbf{b}} \cdot u
$$

with the electric power and its time derivatives

$$
\mathbf{x}=\left[\begin{array}{lll}
x_{1} & x_{2} & x_{3}
\end{array}\right]^{\mathrm{T}}=\left[\begin{array}{lll}
P_{\mathrm{el}} & \dot{P}_{\mathrm{el}} & \ddot{P}_{\mathrm{el}}
\end{array}\right]^{\mathrm{T}}
$$

as the components of the state vector. The system output

$$
y=\mathbf{c}^{\mathrm{T}} \cdot \mathbf{x} \quad \text { with } \mathbf{c}^{\mathrm{T}}=\left[\begin{array}{lll}
1 & 0 & 0
\end{array}\right]
$$

is given by the electric power $P_{\mathrm{el}}$ as a measurable quantity.

The system in Eqs. (4) and (6) will be transformed in such a way that it is possible to predict an optimized future input signal on the basis of a temporally varying reference trajectory. The extended model for the MPC should represent the real process as precisely as required, while being as simple as possible. Under the assumption that the control signal is constant during a sampling period, the MPC makes use of the future output values in a discrete-time form with the discretetime system matrix

$$
\mathbf{A}_{k}=e^{\mathbf{A} T}
$$

and the discrete-time control input vector

$$
\mathbf{b}_{k}=\int_{0}^{T} e^{\mathbf{A} \tau} \mathbf{b} \mathrm{d} \tau \quad \text { with } e^{\mathbf{A} \tau} \approx \sum_{i=0}^{10} \frac{\mathbf{A}^{i} \tau^{i}}{i !} .
$$

To avoid memory overload during the computation, the matrix exponential is approximated with a truncated series expansion of tenth order. Note, the variable $T$ is an integer multiple of the sampling time for data acquisition at the test rig. Hence, the predicted system states are given by

$$
\mathbf{x}_{k+1}=\mathbf{A}_{k} \mathbf{x}_{k}+\mathbf{b}_{k} u_{k} .
$$

Applying (9) recursively and accounting for the output definition according to (6), the discrete-time output is given by

$$
\begin{aligned}
y_{k} & =\mathbf{c}^{\mathrm{T}} \mathbf{x}_{k} \\
y_{k+1} & =\mathbf{c}^{\mathrm{T}} \mathbf{x}_{k+1}=\mathbf{c}^{\mathrm{T}}\left(\mathbf{A}_{k} \mathbf{x}_{k}+\mathbf{b}_{k} u_{k}\right) \\
\vdots & \\
y_{k+M} & =\mathbf{c}^{\mathrm{T}} \mathbf{x}_{k+M}=\mathbf{c}^{\mathrm{T}} \mathbf{A}_{k}^{M} \mathbf{x}_{k}+\mathbf{c}^{\mathrm{T}} \sum_{j=0}^{M-1} \mathbf{A}_{k}^{j} \mathbf{b}_{k} u_{k+M-j} .
\end{aligned}
$$

The resulting set of difference equations according to (10) can be given by the stacked vector notation

$$
\tilde{\mathbf{y}}_{k+1}=\tilde{\mathbf{A}} \mathbf{x}_{k}+\tilde{\mathbf{B}} \tilde{\mathbf{u}}_{k}
$$

with the modified system matrix

$$
\tilde{\mathbf{A}}=\left[\begin{array}{c}
\mathbf{c}^{\mathrm{T}} \mathbf{A}_{k} \\
\mathbf{c}^{\mathrm{T}} \mathbf{A}_{k}^{2} \\
\vdots \\
\mathbf{c}^{\mathrm{T}} \mathbf{A}_{k}^{M}
\end{array}\right]
$$


and the modified input matrix

$$
\tilde{\mathbf{B}}=\left[\begin{array}{cccc}
\mathbf{c}^{\mathrm{T}} \mathbf{b}_{k} & 0 & \ldots & 0 \\
\mathbf{c}^{\mathrm{T}} \mathbf{A}_{k} \mathbf{b}_{k} & \mathbf{c}^{\mathrm{T}} \mathbf{b}_{k} & \cdots & 0 \\
\vdots & \ddots & \ldots & \vdots \\
\mathbf{c}^{\mathrm{T}} \mathbf{A}_{k}^{M-1} \mathbf{b}_{k} & \cdots & \mathbf{c}^{\mathrm{T}} \mathbf{A}_{k} \mathbf{b}_{k} & \mathbf{c}^{\mathrm{T}} \mathbf{b}_{k}
\end{array}\right] .
$$

The vector of the system input

$$
\tilde{\mathbf{u}}_{k}=\left[\begin{array}{llll}
u_{k} & u_{k+1} & \cdots & u_{k+M-1}
\end{array}\right]^{\mathrm{T}}
$$

includes the current and the future inputs for a collection of $M$ time steps.

\section{Model Predictive Control}

\section{A. Control Design}

MPC approaches are widely applicable for many technical processes, especially for processes with a well-known current and future reference trajectory [11]. Here, the reference trajectory over a time window of length $M$ is summed up in

$$
\tilde{\mathbf{y}}_{\mathrm{d}, k+1}=\left[\begin{array}{lll}
y_{\mathrm{d}, k+1} & \cdots & y_{\mathrm{d}, k+M}
\end{array}\right]^{\mathrm{T}},
$$

where a continuous-time variation of $y_{\mathrm{d}}(t)$ is given by a piecewise defined seventh-order Bernstein polynomial [1]. By solving an offline optimization problem, the future input signals will be determined. Therefore, a quadratic cost function

$$
J=\frac{1}{2}\left[\left(\tilde{\mathbf{y}}_{k}-\tilde{\mathbf{y}}_{\mathrm{d}, k}\right)^{\mathrm{T}} \mathbf{Q}\left(\tilde{\mathbf{y}}_{k}-\tilde{\mathbf{y}}_{\mathrm{d}, k}\right)+\tilde{\mathbf{u}}_{k}^{\mathrm{T}} \mathbf{R} \tilde{\mathbf{u}}_{k}\right]
$$

with the weighted, quadratic deviation of the future output signal $\tilde{\mathbf{y}}_{k}$ from its reference signal $\tilde{\mathbf{y}}_{\mathrm{d}, k}$ is defined. Furthermore, the differences of the current and the future control signal $\tilde{\mathbf{u}}_{k}$ are penalized to prevent excessively large variations. For that purpose, the matrix

$$
\mathbf{R}=\eta \mathbf{I}+\mathbf{T}^{\mathrm{T}} \mathbf{N} \mathbf{T}
$$

is defined with $\eta>0$ as a separate penalization for the absolute values of the control inputs and $\mathbf{N}$ as a weighting matrix for their variations. With the help of the Toeplitz matrix

$$
\mathbf{T}=\left[\begin{array}{ccccc}
1 & -1 & 0 & \cdots & 0 \\
0 & 1 & -1 & \ddots & 0 \\
\vdots & \ddots & \ddots & \ddots & \vdots \\
0 & \cdots & 0 & 1 & -1
\end{array}\right],
$$

the difference between the current and the following input is determined. Because of the special, linear structure of the extended model (11), the optimization problem can be determined offline with the necessary optimality condition

$$
\frac{\partial J}{\partial \tilde{\mathbf{u}}}=\mathbf{Q} \tilde{\mathbf{B}}^{\mathrm{T}}\left(\tilde{\mathbf{A}} \mathbf{x}_{k}+\tilde{\mathbf{B}} \tilde{\mathbf{u}}_{k}-\tilde{\mathbf{y}}_{\mathrm{d}, k}\right)+\mathbf{R} \tilde{\mathbf{u}}_{k} \stackrel{!}{=} 0
$$

and by solving the expression (19) for the input vector

$$
\begin{aligned}
\tilde{\mathbf{u}}_{k}= & \left(\tilde{\mathbf{B}}^{\mathrm{T}} \mathbf{Q} \tilde{\mathbf{B}}+\mathbf{R}\right)^{-1} \mathbf{Q} \tilde{\mathbf{B}}^{\mathrm{T}} \tilde{\mathbf{y}}_{\mathrm{d}, k} \\
& -\left(\tilde{\mathbf{B}}^{\mathrm{T}} \mathbf{Q} \tilde{\mathbf{B}}+\mathbf{R}\right)^{-1} \mathbf{Q} \tilde{\mathbf{B}}^{\mathrm{T}} \tilde{\mathbf{A}} \mathbf{x}_{k} .
\end{aligned}
$$

By splitting Eq. (20) in the vector for the feedforward control

$$
\mathbf{S}_{\mathrm{v}}=\left(\tilde{\mathbf{B}}^{\mathrm{T}} \mathbf{Q} \tilde{\mathbf{B}}+\mathbf{R}\right)^{-1} \mathbf{Q} \tilde{\mathbf{B}}^{\mathrm{T}}
$$

and in the feedback control part, the control gain

$$
\mathbf{k}=\left(\tilde{\mathbf{B}}^{\mathrm{T}} \mathbf{Q} \tilde{\mathbf{B}}+\mathbf{R}\right)^{-1} \mathbf{Q} \tilde{\mathbf{B}}^{\mathrm{T}} \tilde{\mathbf{A}}
$$

can be determined. Note, both $\tilde{\mathbf{B}}^{\mathrm{T}} \mathbf{Q} \tilde{\mathbf{B}}$ and $\mathbf{T}^{\mathrm{T}} \mathbf{N T}$ are always positive semi-definite. With a sum of $\eta \mathbf{I}$ and because of the symmetry of the matrix, the inverse matrix included in (21) and (22) always exists. The control law

$$
\begin{aligned}
u_{k} & =\mathbf{e}_{1}^{\mathrm{T}} \cdot \tilde{\mathbf{u}}_{k} \\
& =\mathbf{e}_{1}^{\mathrm{T}}\left(\mathbf{S}_{\mathrm{v}} \cdot \tilde{\mathbf{y}}_{\mathrm{d}, k}-\mathbf{k} \cdot \mathbf{x}_{k}\right)
\end{aligned}
$$

is determined as the actual input signal with the help of the unit vector

$$
\mathbf{e}_{1}=\left[\begin{array}{llll}
1 & 0 & \cdots & 0
\end{array}\right]^{\mathrm{T}}
$$

Hence, the structure of the model predictive control, shown in Fig. 2, is similar to a state-of-the-art state-space control with the difference that the matrices $\mathbf{S}_{\mathrm{v}}$ in the feedforward control (21) and in the feedback gain $\mathbf{k}$ in (22) include the solution of a physically-motivated optimization problem. For the real application, it is only necessary to evaluate the matrices (21) and (22), which implies a straightforward implementation. Furthermore, the included offline optimization saves calculation time to make the system real-time capable even when using low-cost CPUs. To further smoothen the input signal when the control optimization makes use of a larger step size than the data acquisition, a discrete-time linear first-order lag filter $\left(\mathrm{PT}_{1}\right)$ is appended after the evaluation of the input signal $u_{k}$ (with a time constant much smaller than the dominant plant time constants; the resulting model mismatch is captured also by means of the following Kalman filter-based observer). A violation of actuator constraints during the application of this control law is prevented by a trajectory planning with the help of the system's open-loop transfer functions (2) and (3).

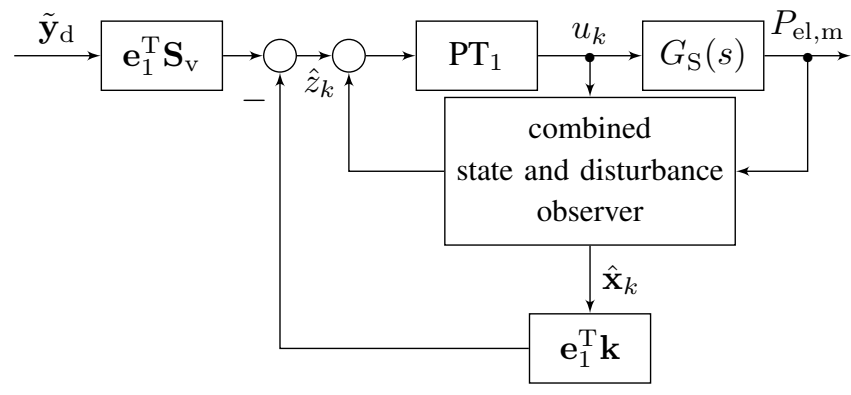

Fig. 2. Structure of the model-predictive control.

\section{B. Estimation of States and Input Disturbance}

For the real-time implementation of the MPC, the current state is required as well as its time derivatives (5). Furthermore, previous work [1] has shown that an additive input disturbance occurs, which should be compensated by the controller. Only the current electric power $P_{\mathrm{el}}$ is measurable, 
so the time derivatives and the additive input disturbance are estimated by a stationary discrete-time Kalman filter. Therefor, the discrete-time state-space representation (9) is extended in

$$
\underbrace{\left[\begin{array}{c}
\mathbf{x}_{k+1} \\
z_{k+1}
\end{array}\right]}_{\mathbf{x}_{\mathrm{e}, k+1}}=\underbrace{\left[\begin{array}{cc}
\mathbf{A}_{k} & \mathbf{b}_{k} \\
\mathbf{0} & 1
\end{array}\right]}_{\mathbf{A}_{\mathbf{e}, k}} \underbrace{\left[\begin{array}{c}
\mathbf{x}_{k} \\
z_{k}
\end{array}\right]}_{\mathbf{x}_{\mathrm{e}, k}}+\underbrace{\left[\begin{array}{c}
\mathbf{b}_{k} \\
0
\end{array}\right]}_{\mathbf{b}_{\mathrm{e}, k}} u_{k}
$$

by the additive input error $z_{k}$ according to a discretized integrator disturbance model to account for a steady-state gain mismatch and temperature-induced variations of the transfer function coefficients in (3). The extended system output

$$
\mathbf{y}_{\mathrm{e}, k}=\left[\begin{array}{ll}
\mathbf{c}^{\mathrm{T}} & 0
\end{array}\right] \mathbf{x}_{\mathrm{e}, k}
$$

will be modified accordingly.

The mathematical approach for the discrete-time Kalman filter

$$
\hat{\mathbf{x}}_{\mathrm{e}, k+1}=\mathbf{A}_{\mathrm{e}, k} \hat{\mathbf{x}}_{\mathrm{e}, k}+\mathbf{b}_{\mathrm{e}, k} u_{k}+\mathbf{h}\left(\mathbf{y}_{\mathrm{e}, k}-\hat{\mathbf{y}}_{\mathrm{e}, k}\right)
$$

includes the extended system (25), shown in Fig. 3.

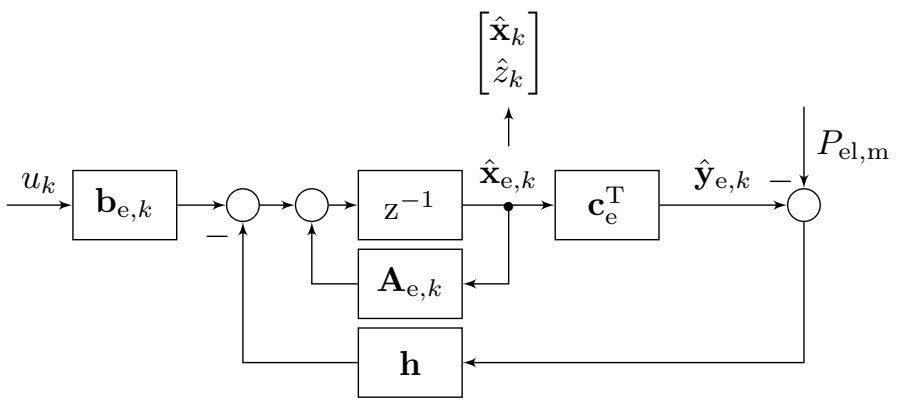

Fig. 3. Structure of the combined state and disturbance observer

Assuming that the system is linear with a constant gain, the optimal observer gain $\mathbf{h}$ is determined by solving the discretetime Riccati equation as the general solution of the stationary Kalman filter [12], [13]. The Matlab function dlqr (...) is employed to minimized a quadratic cost function for which the weighting matrices have to be defined by setting them equal to the covariance matrices of the process and measurement noise.

\section{Simulation Results}

The MPC is tested in a simulation with the 6th order transfer function of the original SOFC plant (2) regarding its capability for trajectory tracking and possible sensitivity against measurement noise. The analysis in [1] already shows that the approximated model (3) leads to good results for the power control with an IMC and a PI controller, which is combined with a dynamic feedforward control. Both of them are usually robust against gain errors or model uncertainties [14], [15]. Now, the MPC design in this paper is compared with the other two control approaches in a simulation with exactly identical boundary conditions. Suitable restrictions of the manipulated variable, e.g. due to the mass flow limitations of the actuators at the test rig, are simulated by added saturation blocks. In an experiment, where the boundary conditions may vary, such a comparison would hardly be possible.

In general, the integral term inside the PI controller, whose structure is shown in Fig. 4, is mainly responsible for the robustness against gain errors. The challenge is to find a compromise between damping and fast transient response. Furthermore, the controller gain needs to be chosen small enough so that the actuator saturations are not triggered, which may result in integrator wind-up phenomena. The IMC has

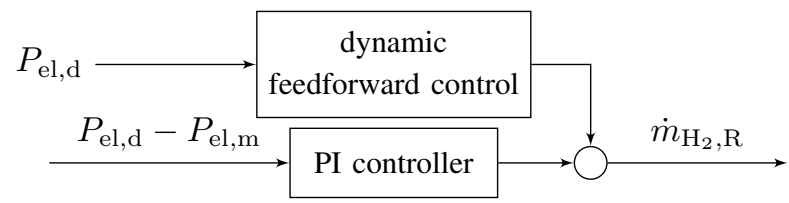

Fig. 4. PI-controller combined with a dynamic feedforward control.

a special structure, see Fig. 5, where the feedback signal comprises the difference between the process and its mathematical model, while alternative controllers usually use the measured or estimated states to determine the feedback signal. Because of the model in the parallel path, which approximates the true system dynamics, the IMC is robust against model uncertainties, e.g. additive input disturbances by design.

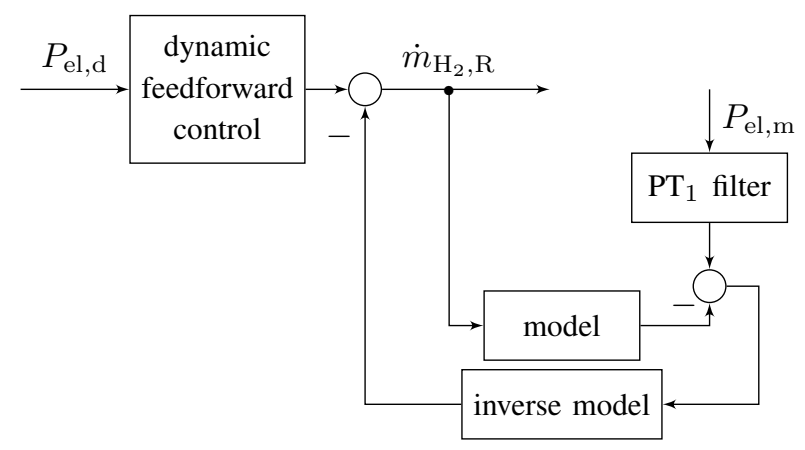

Fig. 5. Structure of the IMC.

To create a simulation as realistic as possible, sensor noise is represented by means of additive Gaussian noise processes with standard deviations according to those of the test rig. The comparison of the results of the simulation of each controller is shown in Fig. 6. It is evident that each controller yields good trajectory tracking, especially in the stationary phase. The significant differences occur in the transient phase.

In correspondence with previous work, the IMC, see Fig. 7, between the desired and the measured trajectory, gives the best result concerning arising errors, which is consistent with the conclusion of the previous work [1]. Over the entire course of time, the IMC has an approximately constant deviation from the desired trajectory. The trajectory of the PI controller shows a slight overshoot before reaching the stationary phase. The MPC reaches the stationary phase a bit more slowly. Nevertheless, each controller delivers a good result. 


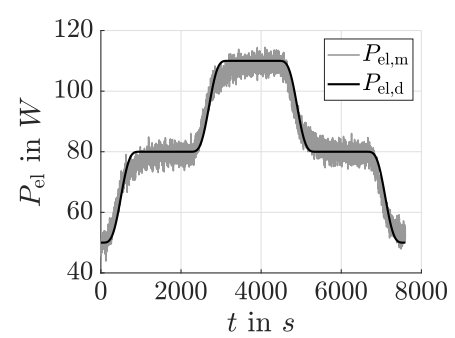

(a) MPC: electric power.

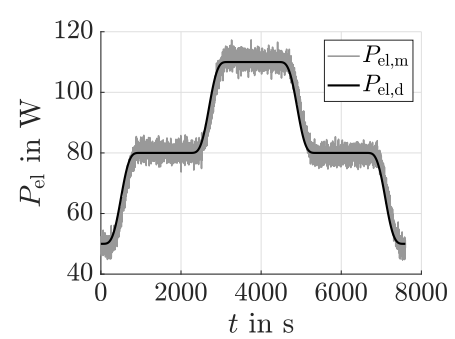

(c) PI: electric power.

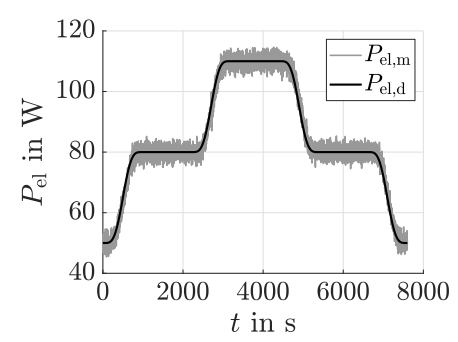

(e) IMC: electric power.

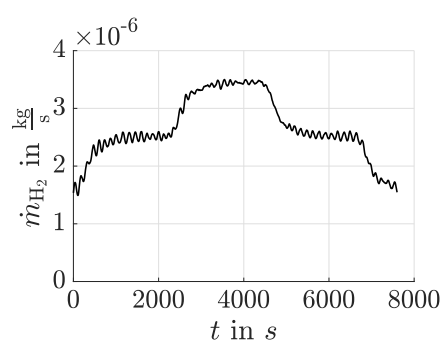

(b) MPC: hydrogen mass flow.

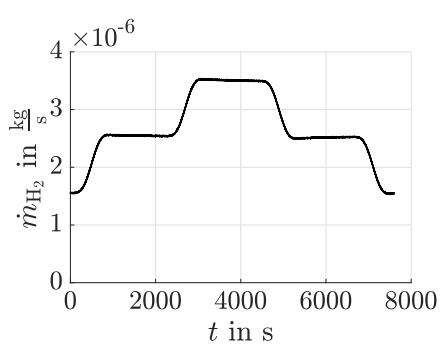

(d) PI: hydrogen mass flow.

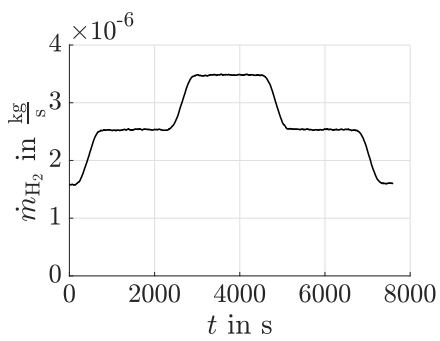

(f) IMC: hydrogen mass flow.

Fig. 6. Simulated input and output signals of the MPC under consideration of a measurement noise compared with the results of an IMC and PI controller.

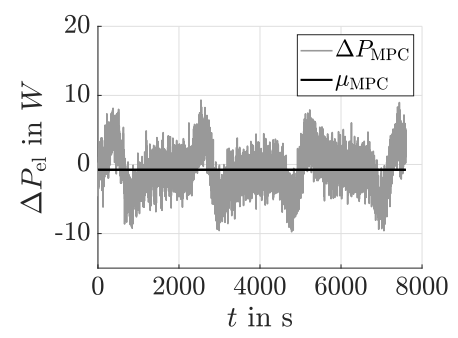

(a) MPC: simulated errors with an (b) PI: simulated errors with an averaverage error of $\mu=-0.767 \mathrm{~W}$ and age error of $\mu=-0.039 \mathrm{~W}$ and a a standard deviation of $\sigma=3.021 \mathrm{~W}$. standard deviation of $\sigma=2.759 \mathrm{~W}$.

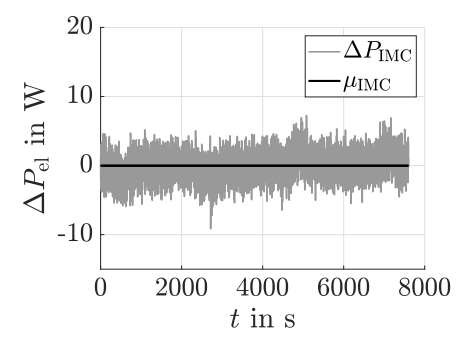

(c) IMC: simulated errors with an average error of $\mu=-0.023 \mathrm{~W}$ and a standard deviation of $\sigma=1.892 \mathrm{~W}$.

Fig. 7. Simulated errors of the MPC under consideration of measurement noise compared with the results of an IMC and PI controller.

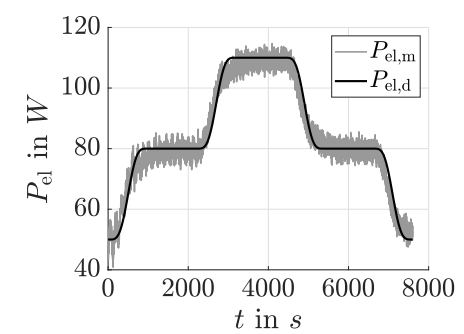

(a) MPC: electric power.

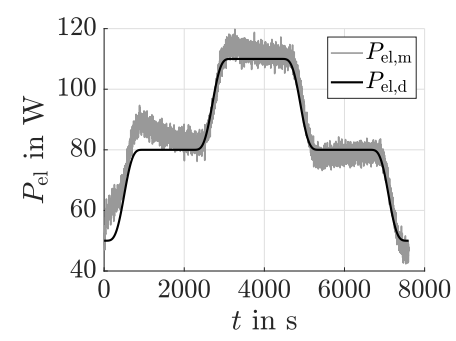

(c) PI: electric power.

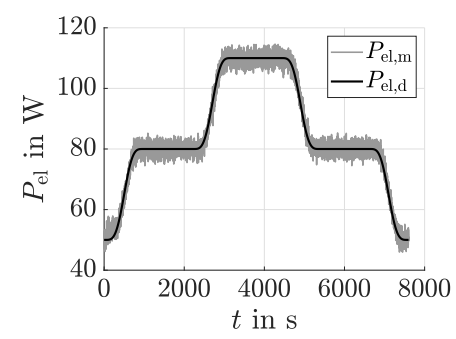

(e) IMC: electric power.

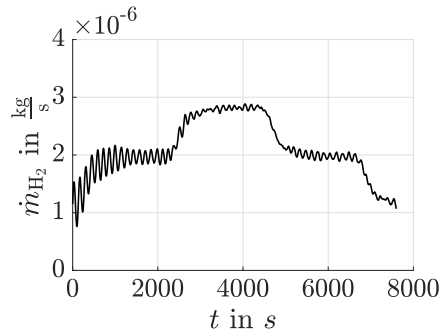

(b) MPC: hydrogen mass flow.

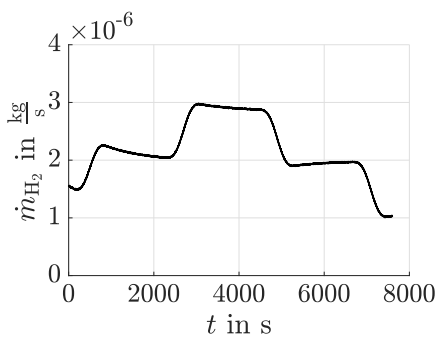

(d) PI: hydrogen mass flow.

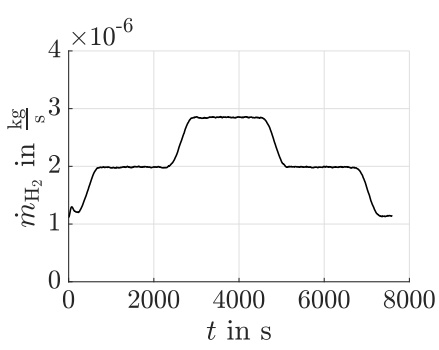

(f) IMC: hydrogen mass flow.
Fig. 8. Simulated input and output signals of the MPC under consideration of $10 \%$ system gain variations and an imitated bad sensor signal with an offset of $10 \mathrm{~W}$ compared with the results of an IMC and PI controller.

\section{Robustness Against Model Uncertainties}

To analyze the behavior of the MPC under the influence of model uncertainty, the measured value $P_{\mathrm{el}, \mathrm{m}}$ was disturbed with a scaling factor to simulate $10 \%$ gain variations of the system. Furthermore, an offset of $10 \mathrm{~W}$ was added to imitate a bad sensor signal. The design of the MPC and the discrete-time Kalman filter remain unchanged for this test. Again, the MPC is compared with the two controllers, PI control and IMC, of previous work [1]. The control accuracy of the compared controllers, shown in Fig. 8 and Fig. 9, has no significant differences from the simulation with the undisturbed values in Sec. IV. Because of the chosen slow time constant, the PI controller has a long response time at the beginning of the trajectory. The integral term guarantees further stationary accuracy, but only after a certain period of time. The MPC continuously has a good trajectory tracking. The controller uses the combined stationary discrete-time Kalman filter to compensate the disturbances. The IMC is able to compensate the disturbance with the help of the inverse model, which is already discussed in Sec. IV, and even has the smallest deviation both in the transient and stationary phase. 


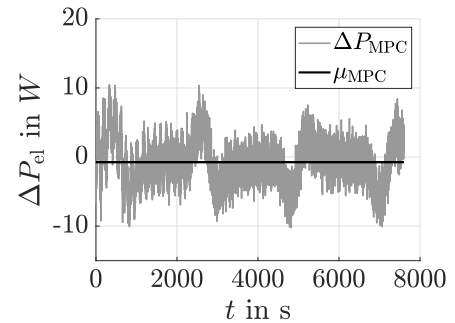

(a) MPC: simulated errors with the average error of $\mu=-0.738 \mathrm{~W}$ and a standard deviation of $\sigma=3.186 \mathrm{~W}$.

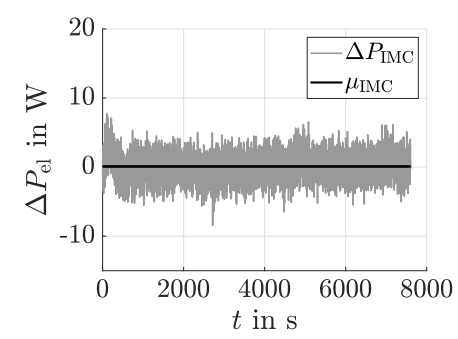

(c) IMC: simulated errors with the average error of $\mu=0.089 \mathrm{~W}$ and a standard deviation of $\sigma=1.840 \mathrm{~W}$.

Fig. 9. Simulated errors of the MPC under consideration of $10 \%$ system gain variations and an imitated bad sensor signal with an offset of $10 \mathrm{~W}$ compared with the results of an IMC and PI controller.

\section{ConClusions And OUtLOOK}

In this paper, an MPC was combined with a discrete-time Kalman filter to control the electric power of an SOFC. Therefor, a discrete-time state-space representation was determined from an approximated third-order lag model based on the measured data. The model was augmented so that the current and future output signals can be calculated in a discrete-time manner, which are required for the MPC design. To derive the control law, a quadratic cost function with different weighting matrices for the penalization of temporal variations of the control input and the differences between the current and future signal were minimized. In a simulation with a nominal and a disturbed process, the MPC was tested with respect to its robustness against model uncertainties. The simulation results were compared with two other controllers, a PI controller combined with a dynamic feedforward control and an IMC, which were already discussed in previous work [1]. In the nominal and the disturbed case, all three approaches lead to a comparable control accuracy. In general, the implementation of MPCs is very simple and intuitive [4], [16]. As shown in this paper, a majority of the implementation effort is to find a linear discrete-time state-space representation as simple as possible, which approximates the dynamics of the system with sufficient accuracy. If this condition is met, the implementation of the MPC itself, which includes the solution of the optimization problem, can be used for every other system. Only the weighting matrices have to be adjusted according to the dynamics. In combination with the stationary discrete-time Kalman filter, the controller is additionally able to compensate the input disturbances. Because of the simple implementation and the robustness against model uncertainties, the MPC is a great alternative as an industrially applicable approach for the power control of SOFCs.

Experiments of previous work [1] show that there is a large gain variation resulting from unavoidable changes of the internal temperature distribution inside the SOFC stack module and from the varying cathode gas temperature. Furthermore, a huge dependency between the electric power and the temperature distribution inside the stack exists, which is not yet considered. For future work, it will be a challenge to determine the system input vector with only one free parameter $b$, see (4), at runtime, which considers this previously described dependency. As a result, a modified feedforward and feedback control gain is expected.

\section{ACKNOWLEDGMENT}

This research was partially funded by the European Union within the European Fund for Regional Development (TBI-V1-256-VBW-090).

\section{REFERENCES}

[1] W. Frenkel, A. Rauh, J. Kersten, and H. Aschemann, "ExperimentsBased Comparison of Different Power Controller for a Solid Oxide Fuel Cell Against Model Imperfections and Delay Phenomena," Algorithms, vol. 13, no. 4, p. 76, 2020.

[2] H.-B. Huo, X.-J. Zhu, W.-Q. Hu, H.-Y. Tu, J. Li, and J. Yang, "Nonlinear Model Predictive Control of SOFC Based on a Hammerstein Model," Journal of Power Sources, vol. 185, no. 1, pp. 338-344, 2008.

[3] J. W. X, X.-J. Zhu, G.-Y. Cao, and H.-Y. Tu, "Predictive Control of SOFC Based on a GA-RBF Neural Network Model," Journal of Power Sources, vol. 179, no. 1, pp. 232-239, 2008.

[4] R. Haber, R. Bars, and U. Schmitz, Predictive Control in Process Engineering: From the Basics to the Applications. John Wiley \& Sons, 2012.

[5] B. Huang, Y. Qi, and A. K. M. M. Murshed, Dynamic Modeling and Predictive Control in Solid Oxide Fuel Cells: First Principle and Databased Approaches. Chichester, UK: John Wiley \& Sons, 2013.

[6] — "Solid Oxide Fuel Cell: Perspective of Dynamic Modeling and Control," Journal of Process Control, vol. 21, no. 10, pp. 1426-1437, 2011.

[7] L. Senkel, Sliding Mode Techniques for Robust Control, State Estimation and Parameter Identification of Uncertain Dynamic Systems. Aachen: Shaker, 2018, PhD Thesis, Chair of Mechatronics, University of Rostock.

[8] A. Rauh, L. Senkel, J. Kersten, and H. Aschemann, "Reliable Control of High-Temperature Fuel Cell Systems using Interval-Based Sliding Mode Techniques," IMA Journal of Mathematical Control and Information, vol. 33, no. 2, pp. 457-484, 2016.

[9] W. Frenkel, "Spatially Distributed Modeling of the Electrochemical Behavior of Solid Oxide Fuel Cells," Master's thesis, Chair of Mechatronics, University of Rostock, 2019.

[10] A. A. Ozdemir and S. Gumussoy, "Transfer function estimation in system identification toolbox via vector fitting," IFAC-PapersOnLine, vol. 50, no. 1, pp. 6232 - 6237 , 2017, 20th IFAC World Congress. [Online]. Available: http://www.sciencedirect.com/science/article/pii/S2405896317315045

[11] E. F. Camacho and C. Bordons, Model Predictive Control. London: Springer, 1999.

[12] M. Papageorgiou, M. Leibold, and M. Buss, Optimierung Statische, dynamische, stochastische Verfahren. Berlin ua Spinger-Verlag, 2015.

[13] R. F. Stengel, Optimal control and estimation. Dover Publications, Inc., 1996.

[14] M. Morari and E. Zafiriou, Robust Process Control. Prentice Hall, Englewood Cliffs, 1989.

[15] S. J. Dodds, Feedback Control. London: Springer-Verlag, 2015.

[16] R. Soeterboek, Predictive Control: A Unified Approach. Prentice-Hall, Inc., 1992. 